\title{
ANALYSIS OF DISPERSION ERRORS IN ACOUSTIC WAVE SIMULATIONS
}

\author{
R. A. C. Germanos ${ }^{\mathrm{a}}$, \\ and L. F. de Souza ${ }^{b}$ \\ ${ }^{a}$ Universidade de São Paulo \\ Departamento de Engenharia Aeronáutica \\ Escola de Engenharia de São Carlos \\ Bairro Centro \\ CP. 13560-970, São Carlos, São Paulo, Brasil \\ gercop@sc.usp.br \\ bUniversidade de São Paulo \\ Departamento de Matemática Aplicada e Estatística \\ Instituto de Ciências Matemáticas e de Computação \\ Bairro Centro \\ CP. 13560-970, São Carlos, São Paulo, Brasil \\ lefraso@icmc.usp.br
}

\begin{abstract}
The governing equations of the acoustic problem are the compressible Euler equations. The discretization of these equations has to ensure that the acoustic waves are transported with non-dispersive and non-dissipative characteristics. In the present study numerical simulations of a standing acoustic wave are performed. Four different space discretization schemes are tested, namely, a second order finite-differences, a fourth order finitedifferences, a fourth order finite-differences compact scheme and a sixth order finite-differences compact scheme. The time integration is done with a fourth order Runge-Kutta scheme. The results obtained are compared with linearized analytical solutions. The influence of the dispersion on the simulation of a standing wave is analyzed. The results confirm that high order accuracy schemes can be more efficient for simulation of acoustic waves, especially the waves with high frequency.
\end{abstract}

Keywords: Acoustic wave, dispersion error, compressible flows, standing wave, finite-difference method, high order methods.

\section{NOMENCLATURE}

disturbance amplitude, $\mathrm{m}$

speed of sound, $\mathrm{m} / \mathrm{s}$

function

derivative function

wavenumber, $\mathrm{m}$

modified wavenumber, $m$

reference length, $\mathrm{m}$

number of points per wavelength

pressure, $\mathrm{N} / \mathrm{m} 2$

time, $\mathrm{s}$

free stream velocity, $\mathrm{m} / \mathrm{s}$

dimensionless velocity, $\mathrm{m} / \mathrm{s}$

cartesian coordinates, $\mathrm{m}$

\section{Greek symbols}

$\alpha \quad$ wave number, $1 / \mathrm{m}$

$\Delta x \quad$ mesh spacing

$\gamma \quad$ dimensionless ratio of specific heats

$\rho$ density, $\mathrm{kg} / \mathrm{m}^{3}$

$\omega \quad$ wave frequency, $1 / \mathrm{s}$

\section{Subscripts}

0 temporal mean flow

$\infty$ free stream

\section{INTRODUCTION}

The engineering research and design requirements of today pose great challenges in computer simulation to engineers and scientists who are called on to analyze phenomena in continuum mechanics. The research area of Computational Aeroacoustics (CAA) is an emerging research area. Its major objective is to help the aerospace industry to drastically reduce aircraft noise. There is also an increased interest in other areas such as automobile noise control. As an extension of Computational Fluid Dynamics (CFD), CAA inherits up to date achievements of CFD, nevertheless, it differs from the traditional CFD research in certain ways. Numerical simulations of these phenomena call for computational methods better suited for accurate and efficient calculation of large scale time dependent problems; and most importantly, a better understanding of the influence of the numerical method over the entire physical wavenumber space is indispensable. This involves improvements in design and analysis of both the basic discretization and numerical boundary conditions. One of the challenges in the CAA area is the accurate calculation of the phenomena, especially in the presence of shock waves. The CAA requires numerical schemes of high accuracy, low dispersion and almost non-dissipation. The need for highresolution discretization methods is a well-known factor in CAA. This is because the numerical study of aeroacoustic requires the correct representation of a large range of spatial and time scales.

In the current work the acoustic waves phenomena was adopted. A very good theory of acoustic waves can be found in Morse and Ingard, 1968, and Tam, 1995, gives a good explanation of the challenges and perspectives in CAA. Tam and Webb, 1992, studied dispersion-relationpreserving finite difference schemes for computational acoustic. Linearized Euler equations were adopted for the numerical model. They emphasize that in uniform mean flow, one has to assure that these partial differential equations support three types of waves: the acoustic, the entropy and the vorticity waves. All three waves are nondispersive and non-dissipative. The difference between them is that, the acoustic waves propagate with the speed of sound, and the others propagates with the speed of the main flow. Because of this characteristic, one has to assure that the numerical results in acoustic simulation are free of dispersion and dissipation. In order to obtain these results, 
Tam and Webb, 1992, developed a finite difference approximation in a way that the Fourier transform is preserved. They obtained an optimized fourth-order explicit approximation with a stencil of 7 points. It was shown for this kind of study that the obtained approximation is better that explicit sixth order approximation, for this kind of study. They also optimized the time discretization, and showed outflow boundary conditions that are "transparent" to the outgoing disturbances. The results obtained by them were very good, showing that non-dispersive methods are important for numerical acoustics.

Vanhille and Pozuelo, 2000, simulate a finite but moderate amplitude standing acoustic wave, using Lagrangian coordinates. In their numerical model a third order partial derivative was obtained. For this derivative a finite-difference scheme of fifth order of truncation error was developed, since the role of this derivative was very important for the formation of the nonlinear standing wave. Their numerical method was validated by comparison with an analytical model. Their results showed the efficiency and the limits of the developed code.

A semi-implicit method for acoustic waves in low Mach number simulations is presented in Wall et all., 2002. The advantage of their proposed method is that the time step is limited only by the convective CFL condition. Their method is second order accurate, both in time and space. An analysis of their results showed that the waves simulated had an average dispersion error of $5 \%$. This was considered by them as not an excessive dispersion error. Their main result is on the gain in computational efficiency, obtained with the semi-implicit method, resulting in a factor of 15 reduction about, as compared with an explicit method.

Spectral methods can be used to assure that all relevant scales are captured, but high order finite difference is also able to represent short length scales with good accuracy. Lele, 1992, emphasizes the importance of using high order methods schemes for first and second derivatives. Mahesh, 1998, presents high order finite difference schemes, introducing a method that, using the same stencil is more accurate than the standard Padé schemes. The disadvantage of his method is that it requires the solution of first and second derivatives simultaneously. Souza et al., 2002 and Souza et al., 2005, used high order compact methods for transition phenomen problems. In these investigated it was studied the propagation of the Tollmien-Schlichting waves in incompressible flows.

Ekaterinaris, 1999 developed a compact high order implicit method to study aeroacustics and twodimensional Euler equation. His results in Aeroacoustics showed a good agreement with the exact solution, and the results with the two-dimensional Euler equation showed that the proposed method presents good results, lowering the total computational time for the simulations when compared with other schemes for the same simulation.

Hixon, 2000, using the algebraic manipulations, proposed a compact finite difference scheme with eighth order accuracy, using 3 points stencil for the simulations of acoustic waves. The main advantage of the proposed method is that one can use the scheme for the points near the boundaries. Results are shown to illustrate the functionality of the method.

Ashcroft e Zhang, 2003, extend the factorization concept proposed by Hixon to a broads class of compact schemes using a more general derivation strategy. Rather than using the algebraic manipulations proposed by Hixon, developed an approach the combines Fourier analysis with the notion of a numerical wavenumber. Two schemes were used, one forward and one backward, giving the optimized prefactored compact scheme. The sum of the schemes recover the original central compact scheme. Their results showed the efficiency of the proposed methods in acoustic waves simulations.

Bogey e Bailly, Bogey showed finite difference schemes with high order accuracy, optimized for acoustic waves simulations, with low numerical dispersion and dissipation, even using 4 points per wavelength. They showed also selective filters that can be used to eliminate spurious oscillations. Runge-Kutta schemes were also studied in their paper, and they showed a sixth order scheme that presented numerical stability with CFL number of 1.98, that represents a gain in the computational time. Other proposed finite difference schemes for numerical simulation of linear acoustic waves can be found in Thomas, 1993; Zingg, 1993; Lockard 1994.

Most sound waves behave as linear waves since they produce pressure fluctuations in air that are very small. A linear waves travels through a medium such as air or water. Fluids such as these can be thought of as consisting of a large number of "particles", each of which consists of a vast number of molecules. Eachof these particles moves as the wave travels through and it passes the disturbance on to its neighbors. However, these small parts of the medium do not travel with the wave. Waves transfer energy without transferring matter.

In the current work, the focus is on the evaluation of discretization error. The tests involved the simulation of one-dimensional standing wave in a periodic domain. Standing wave may be created from two waves, with equal frequency, amplitude and wavelength, traveling in opposite directions. Using superposition, the resultant wave is the sum of these two waves.

The discretization error was analyzed and tested for different space discretization schemes, namely, a second order finite-differences, a fourth order finite-differences, a fourth order finite-differences compact scheme and a sixth order finite-differences compact scheme. Both centered and non-centered schemes were analyzed.

The paper is organized as follows: in section 2 the formulation for the standing wave is shown. The equations adopted are the Euler equation. The numerical method adopted is shown in section 3 . In the same section an analysis of the spatial discretization of the finite difference methods used is done. In section 4 numerical results for various test cases are presented. The conclusions about the discretization errors on Computational Aeroacoustic are shown in the last section.

\section{FORMULATION}

In the current study, the governing equations are the compressible, isentropic, one-dimensional Euler equations. They consist of the momentum equations for the velocity component $(\mathrm{u})$ in the streamwise direction $(\mathrm{x})$ :

$$
\frac{\partial \rho u}{\partial \mathrm{t}}=-\frac{\partial}{\partial x}\left(\rho \mathrm{u}^{2}+p\right)
$$

and the continuity equation:

$$
\frac{\partial \rho}{\partial \mathrm{t}}=-\frac{\partial \rho u}{\partial x},
$$


where $\mathrm{t}$ is the time, $\rho$ is the density and $p$ is the pressure. Finally $p$ and $\rho$ satisfy the idea law gas for a isentropic flow (Sullivan, 1981):

$$
p=\left(\frac{\bar{p}_{\infty}}{\bar{\rho}_{\infty} \bar{U}_{\infty}^{2}}\right) \rho^{\gamma},
$$

where $\gamma$ is the specific heat ratio. The variables used in the above equations are non-dimensional. They are related to the dimensional variables by:

$$
\begin{aligned}
& x=\frac{\bar{x}}{\bar{L}} ; \quad t=\frac{t \bar{U}}{\bar{L}} ; \quad u=\frac{\bar{u}}{\bar{U}} ; \\
& \rho=\frac{\bar{\rho}}{\bar{\rho}_{\infty}} ; \quad p=\frac{\bar{p}}{\bar{\rho}_{\infty} \bar{U}_{\infty}^{2}},
\end{aligned}
$$

where the terms with an over-bar are dimensional terms, $\bar{L}$ is the reference length, $\bar{U}_{\infty}$ is the free-stream velocity and $\bar{\rho}_{\infty}$ is the density of the undisturbed flow.

We can also decomposed the flow in a temporal mean with a small disturbance:

$$
\begin{aligned}
& u(x, t)=u_{0}+\mathrm{u}^{\prime}, \\
& \rho(x, t)=\rho_{0}+\rho^{\prime}, \\
& p(x, t)=p_{0}+p^{\prime},
\end{aligned}
$$

where the index $\left({ }_{0}\right)$ indicates the temporal mean flow (') and indicates of the small disturbance.

\section{NUMERICAL METHOD}

The Eqs. (1) and (2) are solved numerically using a uniform grid. The number of points used in each simulation depended on the discretization method adopted. Therefore, an efficient selection of the grid points numbers and the accuracy of a code can be evaluated by comparison with analytical solution. Finding general analytical solutions of the Euler equations is not easy due to its non-linearity. Because of this, the waves considered had small amplitudes. Taking this into account, the equations were linearized to obtain an analytical solution. This assumption is realistic if the fluctuations are small. The temporary mean flow adopted was null $\left(u_{0}=0\right)$. With these two assumptions one can obtain the following solutions of the Euler equations (1 and 2):

$$
u^{\prime}(x, t)=A \sin (\alpha x-\omega t)+A \sin (\alpha x+\omega t)
$$

$$
\begin{gathered}
\rho^{\prime}(x, t)=\frac{\rho_{0}}{c}\left[u^{\prime}-2 A \sin (\alpha x+\omega t)\right] \\
p^{\prime}(x, t)=\left(\frac{p_{0}}{\rho_{0}^{\gamma}}\right) \rho^{\gamma},
\end{gathered}
$$

where $\alpha$ is the disturbance wave number in the $\mathrm{x}$-direction, $\omega$ is the disturbance frequency and $A$ is the disturbance amplitude.

The acoustics wave propagation develops from specified initial conditions. In the present computations the analytical solutions at time equal zero were used as initial conditions. These conditions were:

$$
\begin{aligned}
& u^{\prime}(x, t=0)=A \sin (\alpha x), \\
& \rho^{\prime}(x, t=0)=0.0, \\
& p^{\prime}(x, t=0)=0.0 .
\end{aligned}
$$

The time integration of equations (1) and (2) were carried out with a classical $4^{\text {th }}$ order Runge-Kutta scheme. In these equations the spatial derivatives were calculated using a finite difference scheme. At the boundaries a periodic condition was used. In this work four different finite difference schemes were evaluated, namely: second order explicit, fourth order explicit, fourth order compact and sixth order compact. Their formula and their respective truncation error are presented bellow:

Second order explicit derivatives:

$$
f_{i}^{\prime}=\frac{1}{2 \Delta x}\left(\mathrm{f}_{\mathrm{i}-1}+\mathrm{f}_{\mathrm{i}+1}\right) \frac{1}{6} \Delta x^{2} f^{3} .
$$

Fourth order explicit derivatives:

$$
\begin{aligned}
& f_{i}^{\prime}=\frac{1}{12 \Delta x^{2}}\left(\mathrm{f}_{\mathrm{i}-2}-8 \mathrm{f}_{\mathrm{i}-1}+8 \mathrm{f}_{\mathrm{i}+1}-\mathrm{f}_{\mathrm{i}+2}\right) \\
& +\frac{1}{30} \Delta x^{4} \mathrm{f}^{5} .
\end{aligned}
$$

Fourth order implicit (compact) derivatives. To find the values of implicit derivatives a matrix must be solved, where all derivatives in a grid line are solved simultaneously. The system can be obtained using the following equation:

$$
\begin{aligned}
& f_{i-1}^{\prime}+4 f_{i}^{\prime}+f_{i+1}^{\prime}=\frac{3}{\Delta x}\left(\mathrm{f}_{\mathrm{i}-1}+\mathrm{f}_{\mathrm{i}+1}\right) \\
& +\frac{1}{5} \Delta x^{4} \mathrm{f}^{5} .
\end{aligned}
$$

Sixth order implicit (compact) derivatives. Here the derivatives are calculated as in the last scheme, solving a system composed by:

$$
\begin{aligned}
& f_{i-1}^{\prime}+3 f_{i}^{\prime}+f_{i+1}^{\prime}= \\
& \frac{1}{12 \Delta x}\left(\mathrm{f}_{\mathrm{i}+2}+28 \mathrm{f}_{\mathrm{i}+1}-28 \mathrm{f}_{\mathrm{i}-1}-\mathrm{f}_{\mathrm{i}-2}\right) \\
& -\frac{1}{28} \Delta x^{6} \mathrm{f}^{7} .
\end{aligned}
$$


A Fourier analysis of the finite-difference methods adopted in the current study was performed. This analysis and the notion of the modified wavenumber provides a convenient means of quantifying the error associated with the differencing schemes. Mahesh, 1998, gives a good explanation of the modified wavenumber considering the test function $f=e^{i k x}$ on a periodic domain, where $\mathrm{k}$ is the wavenumber. Discretize this function on a domain of length $2 \pi$, using a uniform mesh of $N$ points. The mesh spacing is therefore giving by $h=2 \pi / N$. The exact value of the first derivative of $f$ is $i k e^{i k x}$. However, the numerically computed derivatives will be of the form $i k^{\prime} e^{i k x}$. The $k^{\prime}$ is the modified wavenumber.

The $k$ is the modified wavenumber. Figure (1) shown the plot of the modified wavenumberk against wavenumber $k$. In this figure the precision of the different schemes can be compared. The wavenumber was normalized by $k_{\max }=\pi / \Delta x$. According to Lele, 1992, the difference of the modified wavenumber to the exact value is associated with the dispersion error. The wavenumber is related to the number of points $N$ per wavelength by:

$$
k=\frac{2}{N-1} .
$$

In Fig. (2) the plots of phase speed against wavenumber of the analyzed difference schemes are shown. These plots were obtained by considering exact time advancement of the advection equation:

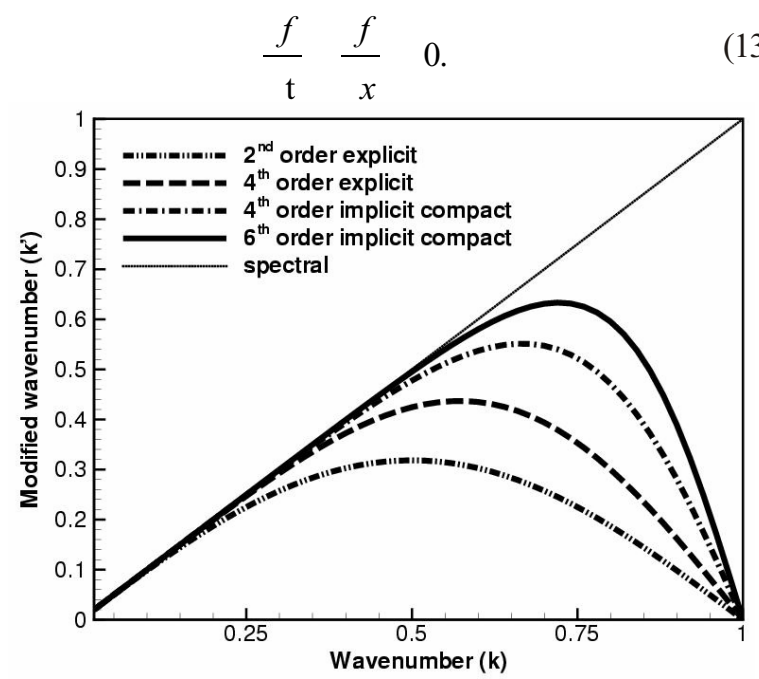

Figure 1. Modified wavenumber $k^{\prime}$ versus wavenumber (k) for first derivative approximations.

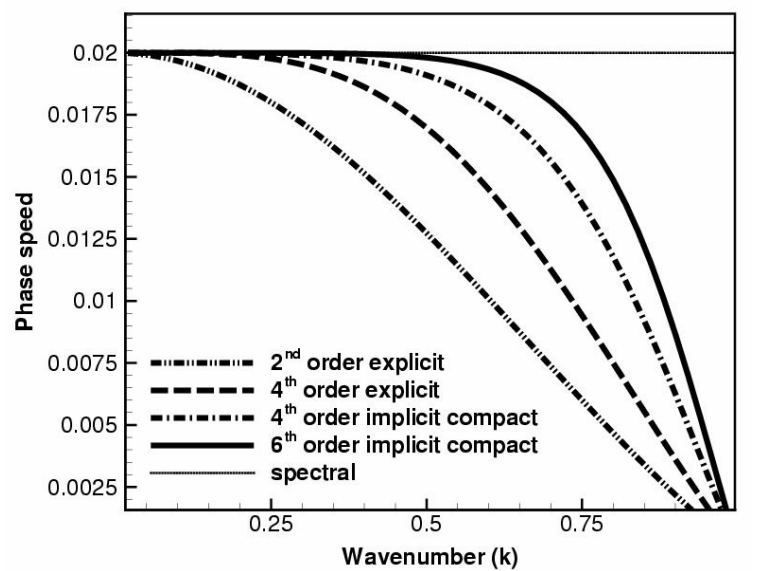

Figure 2. Phase speed versus wavenumber (k) for first derivative approximations.
The next section presents results of a standing wave simulation of infinitesimal amplitude and the results were related with the modified wavelength and phase speed plots.

\section{NUMERICAL RESULTS}

The propagation of standing acoustic waves with a small amplitude was used to analyze the numerical method. The number of points used for discretization of the mesh interferes directly in the precision of the amplitude and phase speed waves, generating a numerical error. This error is called of dispersion error. The study of this error is very important for problems with two or three dimensions, where the computational cost is very high. With this analysis, one can choose the number of points for which the dispersion error is negligible. In this work, the second and fourth order explicit finite-difference methods and the fourth and sixth order compact finite-difference methods were analyzed.

The parameters for the temporary mean flow adopted were those at sea level according to the International Standard Atmosphere. The initial amplitude $A$ of the perturbation was approximately $10^{-4} \mathrm{~m}$ and the phase speed $c$ was $340.21 \mathrm{~m} / \mathrm{s}$. The wavenumber $\alpha$ of the disturbance selected for the simulation was $2 \pi$. The numerical results were simulated for over 100 time periods. The spatial domain extended from $x=0$ to $x=1$.

In the dispersion error analysis a very small time step was adopted. This choice assured that the temporal discretization error was much smaller than the space discretization error. The order of the time step adopted was $10^{-6}$. In the simulations meshes with 5,9 and 13 points per wavelength were used in the $x$ discretization. The mesh with 5 points corresponds to the wavenumber value of $k=0.5$ in the Figs. (1) and (2). The 9 points mesh corresponds to the wavenumber value of $k=0.25$ in the same figures, and the mesh with 13 points corresponds to the wavenumber value of $k=0.167$. In the figs. (3) (7), the solid line corresponds the amplitude of the analytical solution and the dotted line corresponds the amplitude obtained with the numerical simulation at time equals 100 periods. The square, diamond and circle symbols correspond to numerical results obtained with mesh discretization of 5,9 and 13 points for wavenumber.

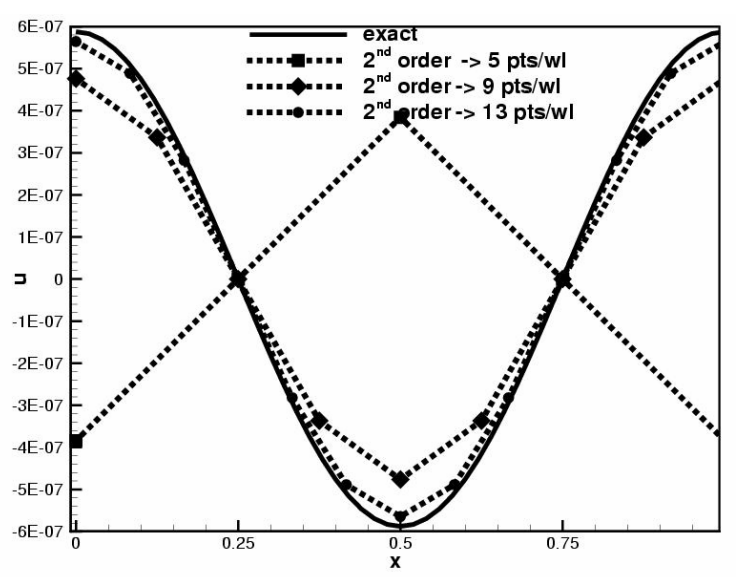

Figure 3. Results of standing wave with second order approximation. 


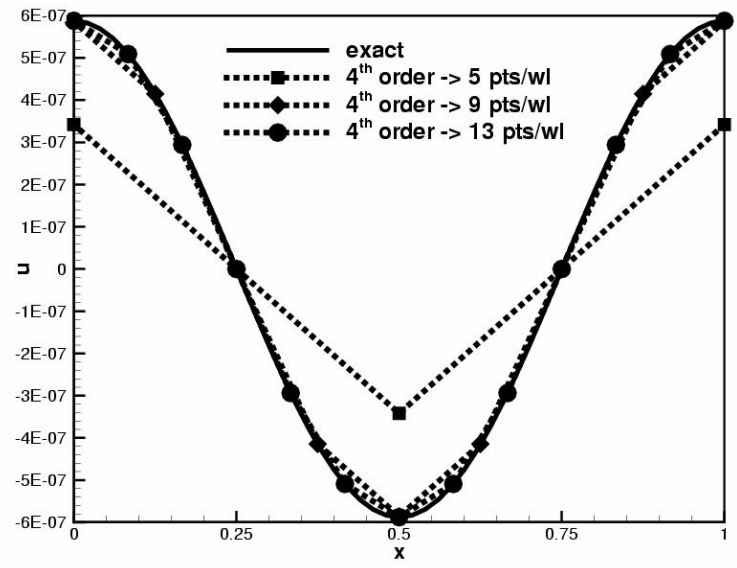

Figure 4. Results of standing wave with fourth order explicit approximation.

Figure (3) shows the numerical results with a second order centered explicit method. The result obtained with the mesh of 5 points shows a large error as compared with the analytical solution. This result can be explained with the help of Fig. (1). There is a difference between the spectral (exact) solution and the second order centered approximation for the wavenumber of $k=0.5$. This difference is directly connected with the dispersion error. This error is reduced increasing the number per wavenumber to 9 and 13 of points. It can be observed that even with wavenumber of $k=0.167$, which that corresponds to 13 points per wavelength one can see a dispersion error. This effect can be understood with the help of Fig. (2), which shows that even with this number of points per wavelength there exists a phase error. The numerical results obtained with the fourth order centered explicit method are presented in Fig. (4). It can be observed that in the result obtained with 5 points per wavelength, the dispersion error is much smaller the one obtained with a second order centered explicit method. The results obtained with 9 and 13 points per wavelength are better and the dispersion error cannot be quantified in this figure. This results are consistent with the graphics shown in Figs. (1) and (2).

Figure (5) shows the numerical results obtained with the fourth order compact method. By comparing Figs. (4) and (5) it can be observed that the result obtained with the fourth order compact method with 5 points per wavelength was more accurate than the result obtained with the fourth order explicit method. For the results obtained with 9 and 13 points per wavelength, the numerical error cannot be quantified by comparing these two figures.

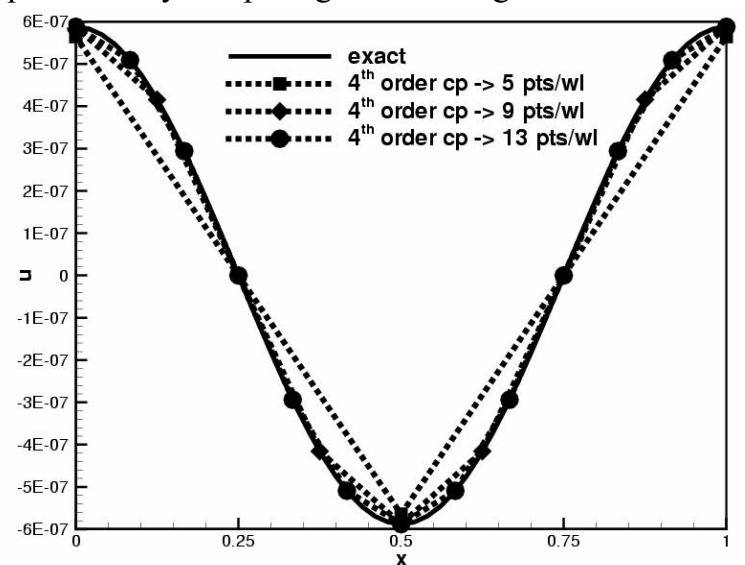

Figure 5. Results of standing wave with fourth order compact approximation.

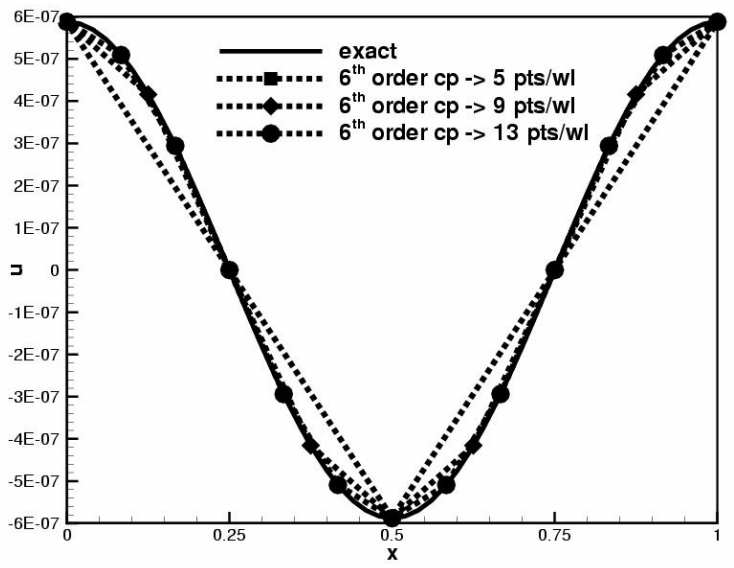

Figure 6. Results of standing wave with sixth order compact approximation.

The last simulation results, using a sixth order compact method is shown in Fig. (6). This method presented the best result for the simulations when compared with the other methods. The amplitude error obtained for the simulation with 5 points per wavelength was very small and cannot be quantified in this figure. The numerical result obtained with 9 and 13 points per wavelength was very good and the dispersion error was almost null. It can be seen in Fig. (2) that the phase error with wavelength of $k=0.5$ is very small for the sixth order compact method. Fig. (6) is consistent with the result.

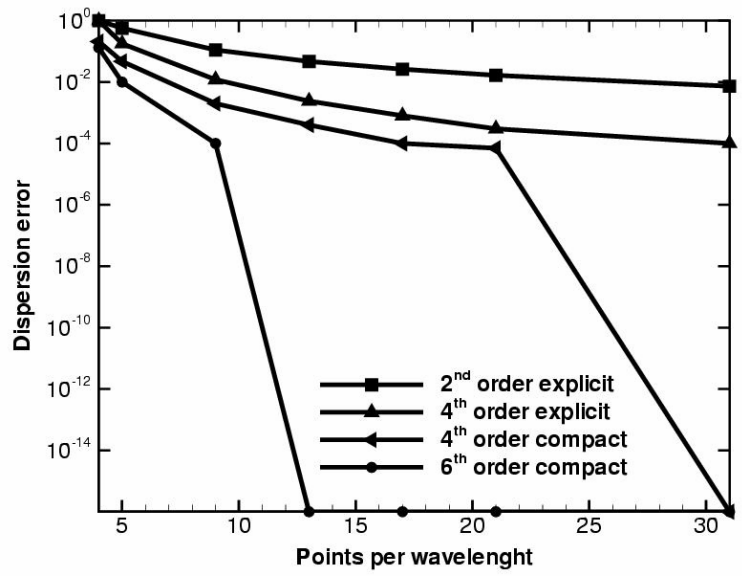

Figure 7. Dispersion of the approximations with different number of points per wavelength.

Figure (7) quantifies the dispersion error obtained in each numerical simulation with different number of points per wavelength. It can be observed that even with a small number of points per wavelength, the sixth order compact is accurate and has low dispersion error. The results obtained with the fourth order compact method shows the advantages in using compact methods. This can be seen by comparing with the fourth order explicit method. This last method shows a large dispersion error if compared with results of the fourth order compact method. The second order explicit method gave the worst results in these simulations. The result obtained with 31 points per wavelength was worse than the result obtained with sixth order compact using 9 points per.

The computational effort of each method was evaluated and the results are shown in table 1 . The simulations were carried out in a Pentium IV $2.8 \mathrm{GHz}$. It 
can be observed that the $6^{\text {th }}$ order compact scheme gives the best result of computational effort, considering the total amount of dispersive error. It can also be seen that explicit schemes presented the worst results. The error obtained with the $2^{\text {nd }}$ order explicit schemes is approximately two hundred larger than with the $6^{\text {th }}$ order compact schemes using 5 points per wavelegth. The last result in this table shows that the error does not increase with the $6^{\text {th }}$ order compact scheme even for large number of periods.

Table 1.Computational effort.

\begin{tabular}{|c|c|c|c|c|}
\hline $\begin{array}{l}\text { Method } \\
2^{\text {nd }} \text { order } \\
\text { explicit }\end{array}$ & $\begin{array}{l}\text { Points per } \\
\text { wavelength } \\
5\end{array}$ & $\begin{array}{c}\text { Periods } \\
1000\end{array}$ & $\begin{array}{c}\text { Error } \\
(\%) \\
18,80\end{array}$ & $\begin{array}{c}\text { Execution } \\
\text { time } \\
00: 55\end{array}$ \\
\hline $\begin{array}{l}2^{\text {nd }} \text { order } \\
\text { explicit }\end{array}$ & 80 & 1000 & 0,1 & $04: 44$ \\
\hline $\begin{array}{l}4^{\text {th }} \text { order } \\
\text { explicit }\end{array}$ & 14 & 1000 & 0,1 & $01: 14$ \\
\hline $\begin{array}{l}4^{\text {th }} \text { order } \\
\text { compact }\end{array}$ & 9 & 1000 & 0,09 & $01: 14$ \\
\hline $\begin{array}{l}6^{\text {th }} \text { order } \\
\text { compact }\end{array}$ & 5 & 1000 & 0,071 & $00: 57$ \\
\hline $\begin{array}{l}6^{\text {th }} \text { order } \\
\text { compact }\end{array}$ & 5 & 10000 & 0,072 & $08: 12$ \\
\hline
\end{tabular}

\section{CONCLUSIONS}

In this work numerical simulation of a standing acoustic wave was performed. The adopted equation was the Euler equation for an isentropic one-dimensional flow. The method adopted was accurate in time, using a fourth order Runge-Kutta scheme, and adopting a very small time step. This assured a very small error in time discretization, allowing a comparison of the dispersion errors of the spatial discretization. The amplitude of the wave was very small in order to present nonlinear behavior. This allows the comparison of the numerical results with the linear analytical solution.

The results showed that the second order explicit method analyzed was dispersive even using many points per wavelength. The results also showed that the fourth order compact method was better than fourth order explicit method analyzed. The execution time was also analyzed showing the advantage in using high order compact schemes. The results confirmed that high order methods are better in wave transport phenomena and should be adopted in acoustical numerical studies.

\section{ACKNOWLEDGEMENTS}

The financial support from FAPESP (State of Sao Paulo Research Support Foundation) -- Grant number 02/09256-3 and 04/07507-4 are greatly acknowledged.

\section{REFERENCES}

Achcroft, G., Zhang X., 2003, Optimized perfactored compact schemes, Journal of Computational Physics, Vol. 190, pp. 459-477.

Bogey, C., Bailly, C., 2004, A family of low dispersive and low dissipative explicit schemes for Engenharia Térmica (Thermal Engineering), Vol. 5 - Nº 01 - July 2006 flow and noise computations, Journal of Computational Physics, Vol. 194, pp. 194-214.

Ekaterinaris, J. A., 1999, Implicit, high-resolution, compact schemes for gas dynamics and aeroacoustics, Journal of Computational Physics, Vol. 156, pp. 272-299.

Hixon, R., 2000, Prefactored small-stencil compact schemes, Journal of Computational Physics, Vol. 165, pp. 522-541.

Lele, S., 1992, Compact finite difference schemes with spectral-like resolution, Journal of Computational Physics, Vol. 103, pp. 16-42.

Lockard, D. P., Brentner, K. S., Atkins H. L., 1994, High accuracy algorithms for computational aeroacoustics, AIAA paper, AIAA-94-0460.

Mahesh, K., 1998, A family of high order finite difference schemes with good spectral resolution, Journal of Computational Physics, Vol. 145, pp. 332-358.

Morse, P. M., Ingard, K. U., 1968, Theoretical Acoustics, McGraw-Hill, New York.

Souza, L. F., Mendonça, M. T., de Medeiros, M. A. F., Kloker, M., 2002, Three dimensional code validatin for transition phenomena, III Escola de Transição e Turbulência.

Souza, L. F., Mendonça, M. T., Medeiros, M. A. F., 2005, The advantages of using high-order finite differences schemes in laminar-turbulent transition studies International Journal for Numerical Methods in Fluids, Vol. 48, pp. 565-582.

Sullivan, D. A., 1981, Historical review of realfluid isentropic flow models, J. Fluids Eng., Vol. 103.

Tam, C. K., 1995, Computational aeroacoustics: Issues and methods, AIAA Journal, Vol. 33(10), pp. 17881796.

Tam, C. K. W., Webb, J. C., 1992, Dispersionrelation-preserving finite difference schemes for computational acoustics, Journal of Computational Physics, Vol. 107, pp. 262-281.

Thomas, J. P., Roe, P. L., 1993, Development of non-dissipative numerical scemes for computational aeroacoustics, AIAA paper, AIAA-93-3382.

Vanhille, C., Pozuelo, C. C., 2000, A high-order finite-difference algorithm for the analysis of standing acoustic waves of finite but moderate amplitude, Journal of Computational Physics, Vol. 165, pp. 334-353.

Wall, C., Pierce, C. D., Moin, P., 2002, A semiimplicit method for resolution of acoustic waves in low Mach number flows, Journal of Computational Physics, Vol. 181, pp. 545-563.

Zingg, D. W., Lomax, H., Jurdens, H., 1993, An optimized finite difference scheme for wave propagation problems, AIAA paper, AIAA-93-0459. 